\title{
Furry hippos and scaly sharks: Knowledge of animal appearance among sighted and blind adults
}

\section{Short Title:}

Blind adults' knowledge of animal appearance

\section{Authors:}

Judy S. Kim a

Giulia V. Elli a

Marina Bedny a

a: Psychological and Brain Sciences

Johns Hopkins University

3400 N Charles St Ames Hall 108, 21210

Classification: Social Sciences, Psychological and Cognitive Sciences

Corresponding Author:

Judy S. Kim

202-701-8767

judyseinkim@gmail.com

Keywords: Blindness, Vision, Language, Appearance, Animals 


\begin{abstract}
How does first-person sensory experience contribute to knowledge? Contrary to the suppositions of empiricist philosophers, people who are blind know about phenomena that cannot be perceived directly, such as color and light. Do blind individuals learn about appearance primarily by remembering sighted people's descriptions of what they see (e.g. "elephants are grey")? How is "verbally-acquired" knowledge about perception represented? We compared knowledge of animal appearance across congenitally blind $(n=20)$ and sighted individuals (two groups, $n=20$ and $n=35$ ). Participants performed a battery of tasks, including ordering animals by size and height and sorting by shape, skin texture, and color (animal names presented in Braille or print). If people who are blind learn primarily from verbal description, blind and sighted groups should share less knowledge about perceptual dimensions that are more difficult to verbalize. We quantified verbalizability by applying the Simpson's Diversity Index to participant labels of shape, texture and color sorting piles. Disagreement across groups was apparent for all dimensions tested, suggesting that visual perception and language convey partially non-redundant information. Contrary to the idea that blind individuals learn primarily from verbal descriptions, blind and sighted groups disagreed most on the dimension that was easiest to verbalize: animal color. Analysis of disagreement patterns suggests that blind individuals infer physical features from other animal properties, such as folk taxonomy and habitat (e.g. bats are shaped like birds but textured like mammals). In the absence of sensory access, structured appearance knowledge is acquired through inference.
\end{abstract}

\title{
Significance Statement
}

We learn about the world through our senses (e.g., we might find out that elephants are grey by seeing one in a zoo). However, sensory experience (e.g., vision) is not always necessary-even people born blind have knowledge about color and light. How is appearance information acquired in the absence of sensory access? A seemingly obvious idea is that blind individuals learn from sighted people's verbal descriptions. We compared blind and sighted people's knowledge of the appearance of common animals and find instead that individuals who are blind infer appearance from other properties (e.g. taxonomy and habitat). In the absence of direct sensory access, knowledge of appearance is acquired primarily through inference, rather than through memorization of verbally-stipulated facts. 


\section{INTRODUCTION}

We learn about the world around us from multiple, redundant sources. We might find out that elephants are grey and have long trunks by observing them in a zoo, hearing people talk about them, reading about them in books, or all of the above. Disentangling the contributions of firstperson sensory experience from verbal communication to knowledge is challenging. Even when first-person sensory experience does not directly convey a particular piece of information, language might reference knowledge originally built from sensory experience. For example, take J.K. Rowling's description of an imaginary creature in "Fantastic Beasts and Where to Find Them":

"The Clabbert is a tree-dwelling creature, in appearance something like a cross between a monkey and a frog. It originated in the southern states of America, though it has since been exported worldwide. The smooth and hairless skin is a mottled green, the hands and feet are webbed, and the arms and legs are long and supple, enabling the Clabbert to swing between branches with the agility of an orangutan. The head has short horns, and the wide mouth, which appears to be grinning, is full of razor-sharp teeth."

Understanding that a Clabbert looks like a cross between a monkey and a frog and is "mottled green" arguably makes use of previous sensory experiences of monkeys, frogs, and the color green.

One approach to disentangling different types of experiences is to compare the knowledge of people with different sensory histories, such as people who are blind from birth and those who are sighted. Empiricist philosophers engaged in thought experiments about blindness (Locke, 1690; Berkeley, 1709; Hume, 1758). Locke proposed, for example, that no amount of explanation or motivation would enable a blind person to understand light and color. He reasoned that a blind person who learns that marigolds are yellow could correctly start describing a marigold as yellow but mistakenly believe that it referred to the texture of a marigold (Locke, 1690).

Empirical studies suggest, however, that blind adults and children do have knowledge about aspects of the world that can only be experienced directly through sight. Young blind children distinguish between different acts of seeing, understanding that one can 'look' at something without 'seeing' it (Landau \& Gleitman, 1985). Blind and sighted adults generate similar features for visual words and judge the same visual verbs to be semantically similar (Lenci et al., 2013, Bedny et al., under review). For example, sighted and blind adults alike distinguish among acts of visual perception along dimensions of duration and intensity (e.g. 'staring' is intense and prolonged, whereas 'peeking' is brief) and among light emission events along dimensions of periodicity and intensity (e.g. 'flash' is intense and periodic, whereas 'glow' is low intensity and stable; Bedny et al., under review).

Contrary to Locke's supposition, blind children and adults also have knowledge of colors. From early in development, blind children understand that colors are properties of physical but not mental objects and can only be perceived with the eyes (Landau \& Gleitman, 1985). Blind adults know that warm colors, like orange and red, are similar to each other but different from cool colors like blue and green (Shepard \& Cooper 1992; Marmor 1978; Saysani, Corballis \& Corballis, 2018). One study found that while semantic similarity judgments for common objects are highly correlated across sighted and blind participants ( $r>0.9$ ), blind individuals are less likely than the sighted to take color into account (Connolly et al., 2007). This is true even for blind individuals 
who correctly report object colors (e.g. bananas are yellow). On the whole, blind individuals turn out to know many things about appearance and vision. However, open questions remain about how blind individuals learn and represent this information.

Blind individuals could learn about imperceptible physical properties by hearing sighted people talk about the things they see. Grammar provides some information-for example, different grammatical constructions such as "see a chair" and "look at a chair" may suggest different word meanings (Gleitman et al., 2005; Landau \& Gleitman, 1985). Many languages, like English, have elaborate vocabularies for colors, light events, and verbs of visual perception (e.g. Majid et al., 2018, Winter, et al., 2018). What is the nature of such verbally acquired knowledge, and exactly how do blind individuals learn from language?

A straightforward prediction of the learn-from-language hypothesis is that blind and sighted people's knowledge about vision and appearance will differ most in cases of low verbalizability. Verbal descriptions of physical appearance are often vague, leaving gaps to be filled in by pragmatics, prior knowledge, and context (e.g. "tall" when it refers to a tree versus a man) (van Deemter, 2009; Solt, 2015, Landau \& Jackendoff, 1993, Krauss \& Fussell, 1992, Westerbeek, Koolen, \& Maes, 2015). Physical dimensions of concrete objects (e.g. size or color) vary continuously, and direct sensory experience provides access to this analog information. Words, on the other hand, refer to discrete aspects of these dimensions (e.g. a cherry and a raspberry are both "red", lions and elephants are both "large") (Talmy 1983; Pinker, 2007; Landau \& Jackendoff, 1993). One might therefore predict that when probed deeply, differences will emerge in what blind and sighted people know about appearance, and that blind and sighted individuals will disagree most about things that are difficult to put into words. The disagreements themselves could also provide insight into how verbally acquired appearance knowledge is organized.

To better understand the origins and structure of appearance knowledge acquired in the absence of first-person sensory experience, the current study examined sighted and blind individuals' knowledge of animal appearance. Animals have multiple physical features, such as size, texture, shape, and color. These features are characteristic of kind and stable over time. We examined knowledge of one dimension that is exclusively visual (i.e. color) as well as multiple dimensions that are in principle accessible via other modalities but for most animals, practically speaking, are perceived through vision (i.e. size, height, shape, and skin texture).

We asked blind and sighted participants to make judgments about animal shape, skin texture, color, size, and height by presenting names of animals in either written print (sighted) or Braille (blind) (Fig. 1). Participants were asked to rank animals by their size and height. Next, participants sorted animals into groups according to their similarity on shape, texture, and color, and were asked to verbally label their sorting piles. Similarity matrices were generated based on the sorting data and compared across groups and dimensions, as well as to a matrix of biological taxonomic similarity. We then quantified the verbalizability of each dimension (texture, shape, color) by calculating Simpson's Diversity Index (SDI). SDI takes into account the number of words used (fewer = more verbalizable) as well as agreement across speakers, and has previously been used to compare verbalizability of different physical dimensions across languages (Majid \& Burenhult, 2014; Levinson \& Majid, 2014; Majid et al., 2018). Participants also performed a set of control sorting tasks (i.e. sorting animals by diet and habitat and sorting objects by typical location). Additionally, participants performed a shape odd-one-out task (i.e. which animal is most different in shape?) and a texture forced choice task (i.e. does this animal have skin, scales, feathers or fur?). Finally, participants rated the overall familiarity of the animals tested to determine whether 
visual access affects the subjective feeling of familiarity. We compared the responses of a blind group and two groups of sighted participants (one tested in the lab and one on Amazon Mechanical Turk).

Figure 1

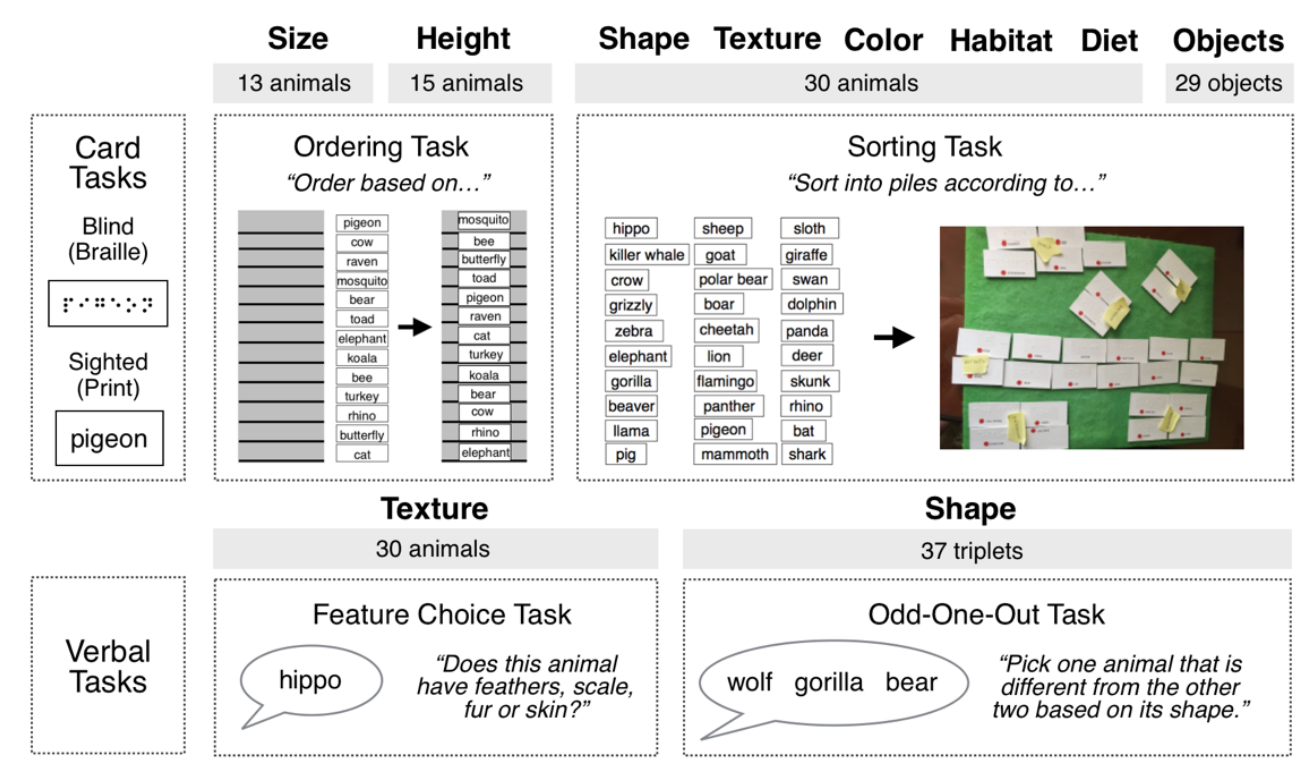

\section{RESULTS}

\section{Familiarity ratings}

Blind and sighted participants' familiarity ratings were highly correlated (Fig. 2, $r=0.82, p<0.001$ ). Blind participants rated animals as overall less familiar than the sighted (sighted: $M=3.26$, $\mathrm{SD}=0.39$, blind: $\mathrm{M}=2.58, \mathrm{SD}=0.55 ; \mathrm{t}(186)=9.87, \mathrm{p}<0.0001)$.

Figure 2

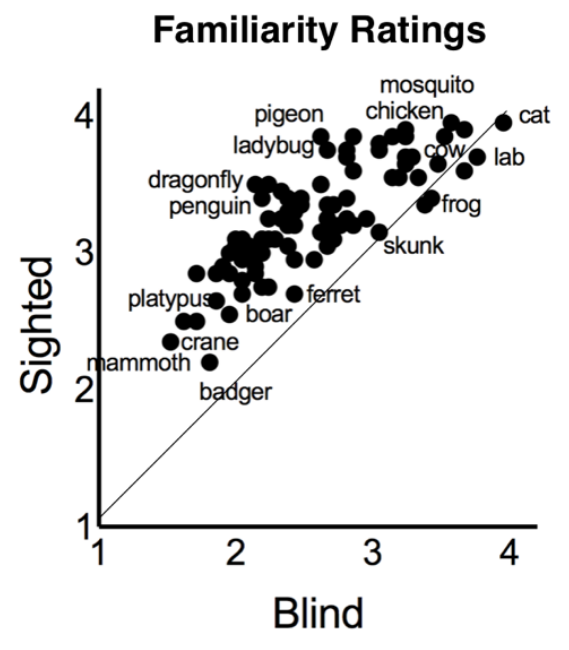




\section{Animal size and height ordering}

Blind participants and two groups of sighted participants (one in the lab and one on Amazon's Mechanical Turk) ordered animals according to their size (13 animals) and height (15 animals), from smallest to largest and shortest to tallest (Fig.1). When rankings were averaged across participants within each group, the resulting orderings were nearly identical across groups, and a subset of blind participants showed high agreement with the sighted (Fig. 3A and 3B). However, on average, blind participants showed significantly lower agreement with sighted MTurk participants, compared to in-lab sighted participants (bar graph in Fig. 3A for size-sighted: $M=80.67 \%, S D=17.29 \%$; blind: $M=49.67 \%, S D=19.4 \%, t(38)=5.33, p<0.0001$; bar graph in Fig. 3B for height-sighted: $M=62.31 \%, S D=12.94 \%$, blind: $M=30 \%, S D=17.45 \%, t(38)=6.65$, $\mathrm{p}<0.0001)$. In particular, relative to the sighted, blind participants made fewer distinctions between individual pairs of animals (right-hand plots in Fig. 3A and $3 B$ ).

Figure 3

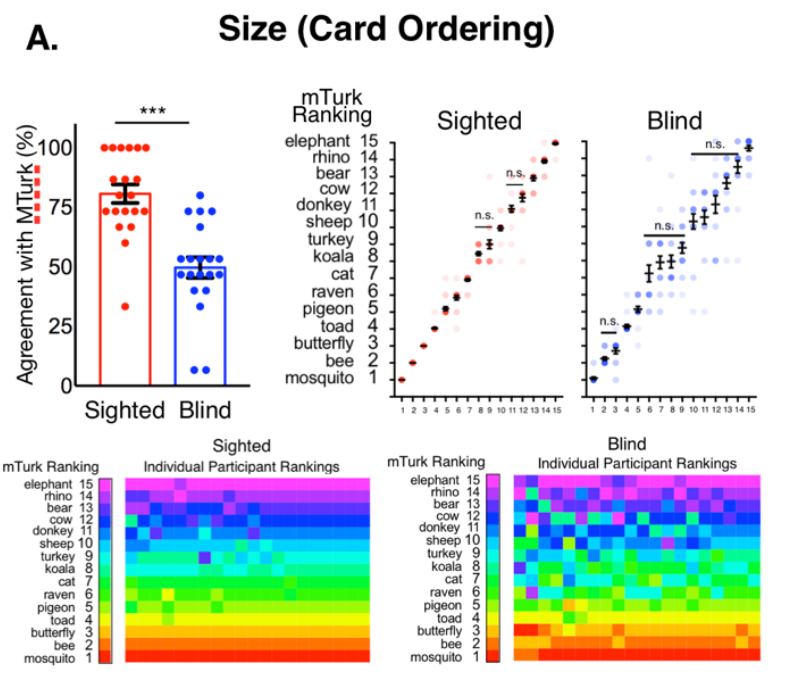

B. Height (Card Ordering)

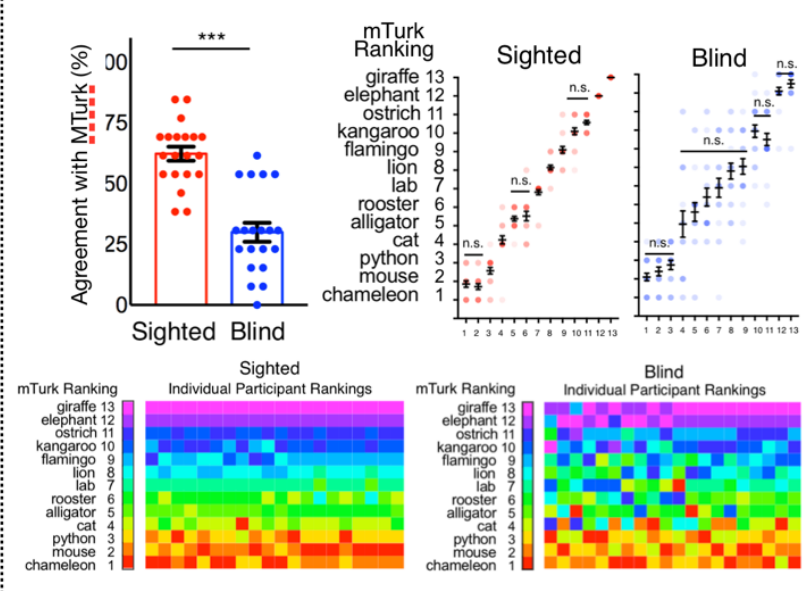

\section{Sorting by shape, texture and color}

Correlations within dimension, within and across groups

To analyze results from the card sorting task ( 30 animals), for each pair of animals, 1 was given if two animals were placed in the same pile and 0 otherwise. Individual subject binary matrices were then averaged across participants within group and task to generate group similarity matrices. 
Figure 4

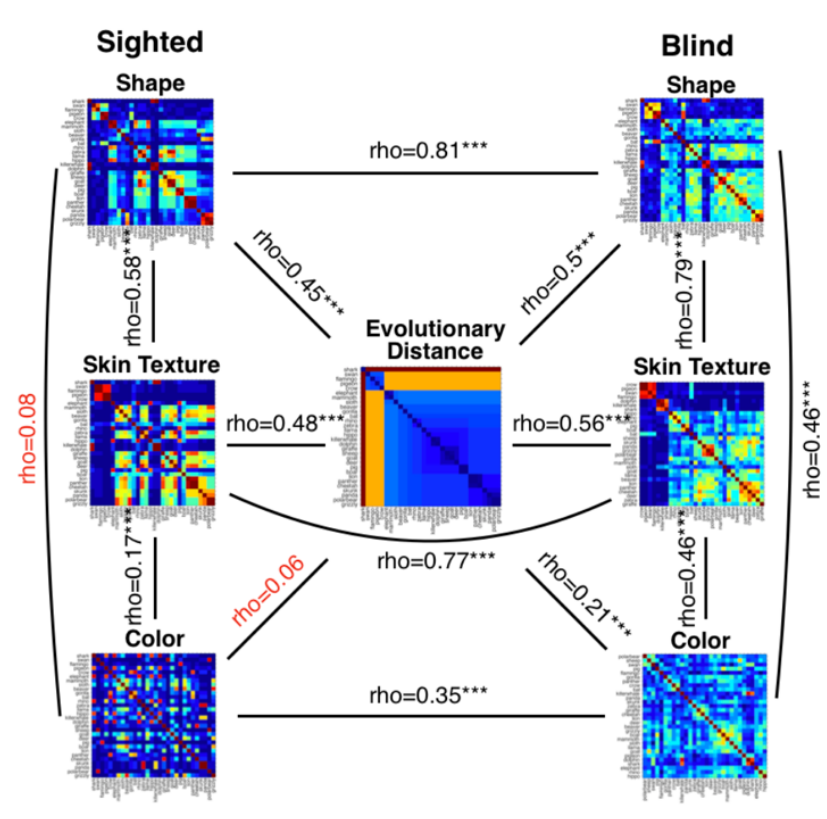

Figure 5

Card Sorting Results

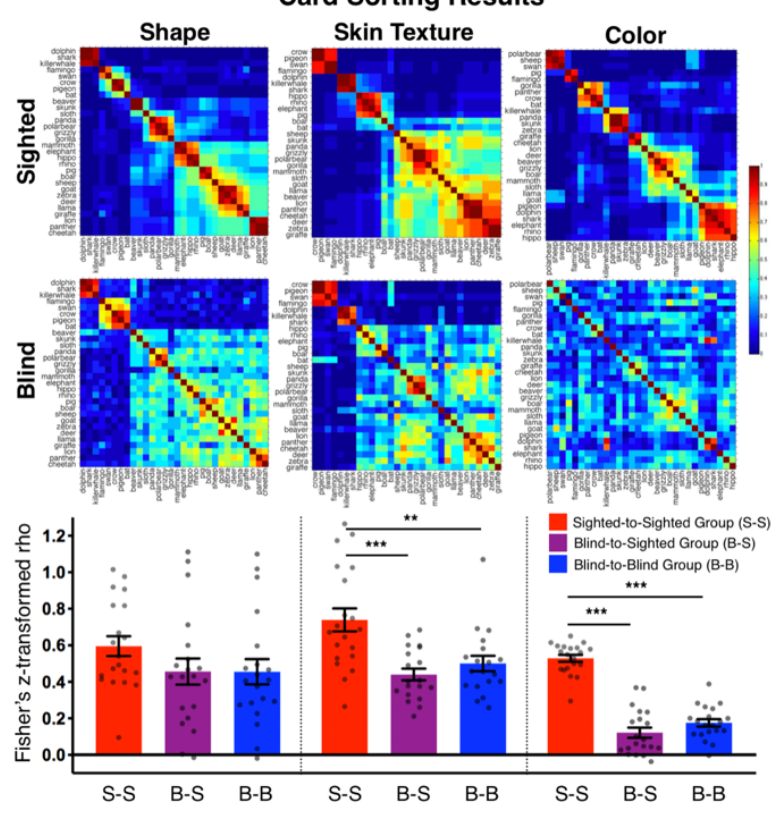

For shape, group similarity matrices were significantly correlated across blind and sighted groups (Fig. 4, rho=0.81, $\mathrm{p}<0.0001$ ). We next correlated individual subjects to the group matrices using a leave-one-subject out procedure. The individual-sighted-to-sighted correlation was slightly but not significantly higher than the blind-individual-to-sighted-group and blind-individual-to-blindgroup correlation (Fig. 5 bar graphs; S-to-S correlation coefficients: $M=0.60, S D=0.24$; B-to-S ztransformed correlation coefficients: $\mathrm{M}=0.46, \mathrm{SD}=0.32$; comparing B-to-S vs. $\mathrm{S}$-to-S: $\mathrm{t}(38)=1.55$, $p=0.13$; B-to-B correlation coefficients: $M=0.46, S D=0.3$; comparing B-to-B vs. $S$-to-S: $t(38)=1.6$, $\mathrm{p}=0.12)$.

Group-wise skin texture matrices were also similar across blind and sighted groups (Fig. 4, rho $=0.77, p<0.0001$ ). However, unlike with shape, individual blind participants' sortings were significantly different from those of sighted participants and more variable within the blind group (Fig. 5 bar graphs; S-to-S correlation coefficients: $M=0.74, S D=0.28$; B-to-S: $M=0.44, S D=0.14$; comparing B-to-S vs. S-to-S: $\mathrm{t}(37)=4.15, \mathrm{p}=0.0002$; $B$-to-B correlation coefficients: $\mathrm{M}=0.5$, $\mathrm{SD}=0.18$; comparing B-to-B vs. S-to-S: $\mathrm{t}(27)=3.11, \mathrm{p}=0.004)$.

Group differences for color sorting were even more pronounced than for skin texture. The correlation between group matrices was smaller, although still significant ( $r h o=0.35, p<0.0001$ ), and blind participants' answers were significantly different from those of the sighted as well as more variable (Fig. 5 bar graphs; S-to-S correlation coefficients: $\mathrm{M}=0.53, \mathrm{SD}=0.09$; B-to-S: $\mathrm{M}=0.12, \mathrm{SD}=0.12$; comparing $\mathrm{B}$-to-S vs. $\mathrm{S}$-to-S: $\mathrm{t}(38)=12.11, \mathrm{p}<0.0001$; $\mathrm{B}$-to-B correlation coefficients: $M=0.18, S D=0.09$; comparing B-to-B vs. $S$-to-S: $t(38)=12.7, p<0.0001)$.

A one-way ANOVA confirmed that the degree to which blind and sighted groups disagreed differed across dimensions (comparing B-to-S across shape, texture, color: $F(2,56)=4.73, p=0.01$ ). Pairwise comparisons further confirmed that group differences were more pronounced for color than for shape or texture (B-to-S for shape vs. texture: $t(37)=0.21, p=0.8$; shape vs. color: $\mathrm{t}(38)=4.39, \mathrm{p}<0.0001$; texture vs. color: $\mathrm{t}(27)=7.5, \mathrm{p}<0.0001)$. 
Visual inspection of the group matrices suggests that for shape and texture, blind and sighted participants made similar groupings based on physical features that covary with taxonomy and habitat. For example, both groups separated aquatic animals (dolphin, shark, killer whale) from birds (pigeon, crow, swan, flamingo) and four-legged land mammals. In contrast, small clusters within the broad taxonomic class of four-legged land animals differed across groups. For instance, when sorting by shape, only sighted participants grouped gorilla, grizzly bear, polar bear, panda, sloth, beaver, and skunk as being distinct from other four-legged animals such as pigs, boars, and sheep. Analogously, when sorting by skin texture, only sighted participants grouped hippo, rhino, elephant, and pig into a distinct sub-category.

For color, sighted formed groups for white (polar bear, sheep, swan), pink (pig, flamingo), black (gorilla, panther, crow, bat), black and white (killer whale, panda, skunk, zebra), yellow (giraffe, cheetah, lion), brown (e.g., deer, grizzly bear), and grey (pigeon, dolphin, shark, elephant, rhino, hippo) animals with high consistency. Blind participants' color sortings, however, did not reveal any such groups.

\section{Correlations with taxonomy (evolutionary distance)}

There was an increase in the across- relative to within- dimension correlations in the blind relative to the sighted group (SI Fig. 1). For the sighted group, all within-dimension correlations were significantly higher than across-dimension correlations (e.g., shape sighted-individual-to-sightedgroup higher than shape-to-texture and shape-to-color). For the blind group, this effect was significant for texture, in the right direction but not significant for shape, and not present for color.

For shape, both blind and sighted group similarity matrices were significantly correlated with evolutionary distance (Fig. 4, sighted: rho $=0.45, p<0.0001$, blind: rho $=0.5, p<0.0001$ ). Correlation coefficients for individual participants' shape sorting matrix correlated to taxonomy similarly showed no group differences (SI Fig. 2, $\mathrm{t}(38)=0.45, \mathrm{p}=0.6$ ). Similarly, for skin texture, both blind and sighted group similarity matrices were significantly correlated with evolutionary distance (Fig. 4, sighted: rho $=0.48, p<0.0001$, blind: rho $=0.56, p<0.0001)$. Individual participants' correlations with taxonomy again did not differ across groups (SI Fig. 2, $\mathrm{t}(37)=0.21, \mathrm{p}=0.8$ ). For color, only the blind group's color similarity matrix was correlated with evolutionary distance (Fig. 4, sighted: rho=0.06, $\mathrm{p}=0.6$; blind: $r h o=0.21, p<0.0001)$. Unlike with shape and skin texture, correlation coefficients for correlation between taxonomy and individual matrices was significantly different across groups (SI Fig. 2, $\mathrm{t}(38)=2.94, \mathrm{p}=0.006$ ).

\section{Verbal labels of sorting groups for shape, skin texture and color sorting}

Verbalizability of sighted participants' descriptions for sorting piles (Simpson's Diversity Index, see Methods for detail) was lowest for shape, followed by skin texture, then color (Fig. 6, shape: $M=0.11, S D=0.03$; skin texture: $M=0.17, S D=0.12$; color: $M=0.47, S D=0.22$; color vs. texture: $t(29)=7.42, p<0.0001$; texture vs. shape: $t(29)=2.51, p=0.018)$. Note that the average number of sorting groups created by sighted participants was comparable across dimensions: shape $=6.8$, texture $=5.1$, color $=7$. 
Figure 6

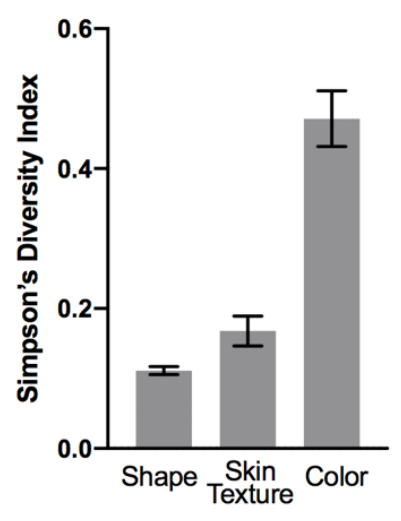

\section{Performance on control sorting tasks}

Correlation between group matrices were high and significant across all control sorting tasks (SI Fig. 3, objects: rho $=0.8, p<0.0001$, habitat: $r h o=0.83, p<0.0001$, diet: rho $=0.78, p<0.0001)$. For sorting objects based on where they are stored, blind participants' answers did not differ from those of the sighted group, although they were more variable (S-to-S correlation coefficients: $M=0.95, S D=0.11$; B-to-S: $M=0.88, S D=0.19$; S-to-S vs. B-to-S: $t(37)=1.36, p=0.18$; $B$-to-B: $M=78$, $\mathrm{SD}=14$; S-to-S vs. $\mathrm{B}$-to-B: $\mathrm{t}(37)=4.11, \mathrm{p}=0.0002)$. For sorting animals by where they live, there were no within- or across-group differences (S-to-S correlation coefficients: $M=0.59, S D=0.11$; $B-$ to-S: $M=0.53, S D=0.16$; S-to-S vs. B-to-S: $t(33)=1.39, p=0.17$; $B$-to-B: $M=0.53, S D=0.15$; S-to-S vs. $B-t o-B: t(33)=1.39, p=0.17)$. Somewhat surprisingly, blind and sighted groups differed in how they sorted animals by diet (S-to-S correlation coefficients: $M=0.57, S D=0.16$; B-to-S: $M=0.36$, $\mathrm{SD}=0.26$; S-to-S vs. B-to-S: $\mathrm{t}(38)=3.04, \mathrm{p}=0.004$; $B$-to-B: $M=0.34, \mathrm{SD}=0.18$; S-to-S vs. B-to-B: $t(38)=4.33, p=0.0001)$.

\section{Shape odd-one-out triplet task}

For the shape odd-one-out task, blind and sighted participants' responses were compared to responses of sighted MTurk participants (i.e., for each triplet, the animal chosen by a plurality of MTurk participants as the odd-one-out was considered the sighted agreed upon answer). Blind participants showed significantly less agreement than the sighted with sighted MTurk participants (Fig. 7A, blind: $M=55.12 \%, S D=10.65 \%$, sighted: $M=74.44 \%, S D=9.22 \%, t(37)=6.07, p<0.0001$ ). On some of the trials where taxonomy was pitted against shape, blind and sighted groups diverged in their answers (e.g., for 'wolf/gorilla/bear', 70\% of sighted participants picked wolf, while $63 \%$ of blind participants chose gorilla, and for 'parrot/giraffe/ostrich', $65 \%$ of sighted said parrot, and $63 \%$ of blind participants answered giraffe).

\section{Skin texture: feature choice task}

For the skin texture feature choice task, answers were scored according to whether they agreed with the following criteria: for reptiles and fish, "scales", "feathers" for birds, "fur" for mammals whose hides are covered in fur or hair (e.g., gorilla, sheep, horse, cat), and "skin" for all other mammals (e.g., dolphin, elephant, pig) (seem Methods for complete list). While there was substantial agreement overall in blind and sighted people's responses, there were also clear differences between groups (Fig. 7B, sighted agreement to criteria: $M=82.83 \%$, SD=4.5\%, blind 
agreement: $\mathrm{M}=69.5 \%, \mathrm{SD}=13.3 \%, \mathrm{t}(38)=4.25, \mathrm{p}=0.0001)$. Inspection of the data suggests that blind and sighted participants' were similarly likely to say that birds have feathers and reptiles have scales (e.g. 100\% of the sighted and $95 \%$ of blind participants said pigeons have feathers and $89 \%$ of blind and $95 \%$ of sighted participants said goldfish have scales). However, blind participants differed from the sighted in classifying mammals according to whether they have skin or fur. A sizeable proportion of blind participants answered that elephant (32\%), rhino (42\%), pig $(68 \%)$, and hippo (42\%) have fur, whereas almost all sighted participants (>95\%) responded that they had skin. Interestingly, blind participants reported that sharks have scales (68\%), while sighted reported that they have skin (75\%). In fact, sharks have fine scales called placoid scales, which are not easily visible with the naked eye.

Figure 7
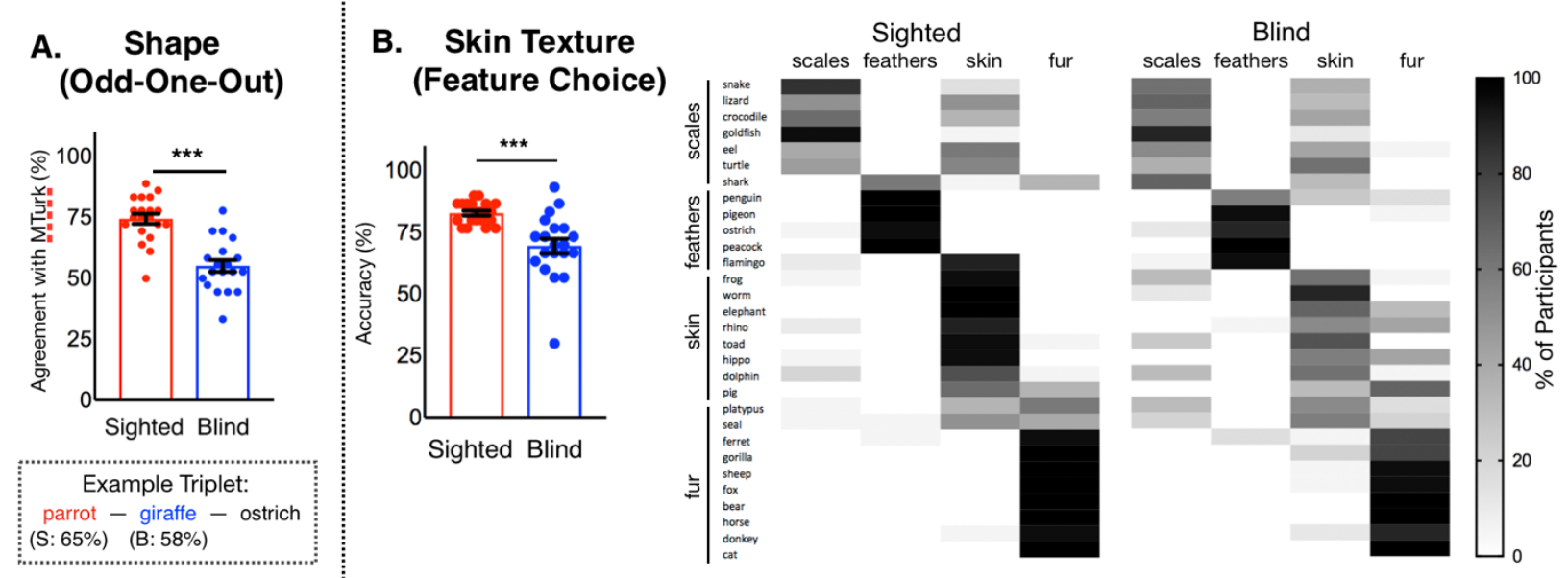

\section{DISCUSSION}

\section{Language is an efficient but incomplete source of information about appearance: Partially shared knowledge of animal appearance among blind and sighted}

The present results show that blind and sighted individuals living in the same culture share a substantial body of information about the shape, texture, height, and size of common animals. This knowledge can be queried with verbal stimuli: judgments of every physical dimension were correlated across individuals (rho>.5), and for sighted participants, much more so within- than across- dimensions (i.e. shape to shape vs. shape to texture). There was considerable agreement between sighted and blind groups, even in cases where the majority of blind individuals are unlikely to have had direct sensory access. For example, 15 out of 20 blind and 19 out of 20 sighted subjects judged elephants to be bigger than rhinos. Participants in both groups reported that birds have feathers and that most land mammals have fur. In the card sorting task, $66 \%$ of the variance for shape and $59 \%$ of the variance for texture was common to sighted and blind groups. For shape, texture, height, and size, blind participants tended to agree amongst themselves and with sighted participants on distinctions between groups of animals, and a subset $(>30 \%)$ of blind participants made judgments that were indistinguishable from those of the sighted. 
Despite substantial shared knowledge, there were differences across groups as well. First, while blind people agreed with the sighted about which animals were most familiar, overall, blind individuals rated animals as less familiar. Disagreement about appearance across groups was most pronounced for color, and apparent even for dimensions such as size, height, shape, and texture, which are in principle available via touch. For size, height, shape, and texture, there was greatest disagreement regarding the appearance of individual animals. For example, when sorting by height and size, blind and sighted participants agreed that elephants and rhinos are larger than bears and cows, and that elephants and giraffes are taller than kangaroos and lions. However, only sighted participants also systematically judged bears to be larger than cows and giraffes to be taller than elephants. Similar effects emerged across all of the tested dimensions.

On the whole, when it comes to appearance, blind and sighted people share knowledge of animal groupings (e.g. fish, birds, reptiles and amphibians, land mammals, sea mammals), knowledge of which animals belong to which groups, and of which physical features are most likely to be observed in each group (e.g., birds have feathers). There is also some shared knowledge about the appearance of individual animals, that is likely acquired from verbal descriptions (e.g. elephants are one of the larger land mammals). However, language and vision are not equivalent sources of information about what things look like (Pinker, 2008; Landau \& Jackendoff, 1993; Rubinstein et al., 2015; Collell \& Moens, 2016). Whether because descriptions do not contain the relevant information or because representations of appearance are not learned from description as easily as they are from perception, representations of appearance developed with and without vision are different.

The current results inform an ongoing discussion about how the source of knowledge affects the nature of the representations formed. A number of recent neuroimaging studies have suggested that blind and sighted people have similar neural representations of object appearance in the ventral occipito-temporal cortex and elsewhere in the brain (e.g. He et al., 2013; Mahon et al., 2009; Peelen et al., 2013; Kitada et al., 2013; van Den Hurk et al., 2017). This neuroimaging work has focused on neural differences between broad categories of objects, such as faces and places. The present results suggest that when it comes to distinctions in the appearance of individual entities within the animal category, representations acquired through language versus visual perception are not indistinguishable. One possibility is that blind and sighted individuals share broad category distinctions but differ on the fine-grained, within-category distinctions. Alternatively, it has recently been suggested that representations of appearance (and shape in particular) are more different across sighted and blind groups for living things than other categories such as artifacts, because in the case of living things, shape cannot be inferred from motor interactions (Bi, Wang \& Caramazza, 2016). Whatever the explanation, blind and sighted people differ in the details of their representations of the surface properties of distal objects.

There is also evidence that relative to the sighted, blind individuals develop more elaborated mechanisms for recognition and categorization through audition, olfaction and touch, showing superior recognition of voices, odors, and Braille letters (Bull et al., 1983, Cuevas et al, 2009, Wong et al., 2011). Together these results suggest that humans develop representations of surface properties of objects that are adapted to their own sensory experience.

\section{Learning about appearance through inference from ontology}

While some of the differences in the knowledge of sighted and blind groups are likely related to the difficulty of describing complex continuous dimensions in words, the present results also 
suggest that description is not the primary source of information about animal appearance for people who are blind. When looking across dimensions, blind and sighted participants disagreed most on the dimension that was easiest to verbalize: color. Sighted subjects' descriptions of their color groups were succinct, typically one or two words, precisely captured how members of a group were similar to each other (e.g. 'black and white' for pandas, killer whale, zebra and skunk), and were common across participants (i.e. groups were labeled as 'white', 'brown', 'pink/red', 'grey', 'black', 'black and white', and 'yellow' by $90 \%$ of sighted participants). Thus, the aspects of color knowledge that were tested in the current study are highly verbalizable. Yet, color was the only dimension for which no single blind participant was indistinguishable from the sighted group, and the correlation between sighted and blind groups was lower than the correlation across dimensions within the blind group. In other words, despite being verbalizable, animal colors are not systematically acquired by people who are blind.

By contrast, shape was the least verbalizable of the tested dimensions. When sighted participants were asked to label their shape-based sorting piles, labels were long, inefficient, and variable across participants, and did not closely mirror how animals were sorted. Descriptions frequently contained features that applied to multiple groups of animals, and therefore failed to capture the similarities unique to the members of a specific group (e.g. 'four legs' or 'large'). Thus in the current study, shape was less verbalizable than color. Consistent with these results, some previous studies suggest that shape is less verbalizable than color across languages (Majid \& Burenhult, 2014; Levinson \& Majid, 2014; see Majid et al., 2018). Yet, blind and sighted participants agreed the most about shape.

Why are blind individuals more likely to agree with the sighted about animal shape than color? It is possible that despite being easier to describe, animal color is talked about less. We are not aware of any evidence for this idea, however. Another explanation, then, can be found in the writings of Locke (1690). He argued that a blind person could learn the shape but not the color of a novel statue from description because blind people have previously experienced shape but not color non-verbally (i.e. through touch). According to this idea, when a sighted person learns that 'a polar bear is white', they can apply the word to their memory of white, whereas blind individuals only have access to the association between the words 'white' and 'polar bear.' By contrast, hearing that something is 'round' is equivalent for sighted and blind individuals.

Locke's inaccessibility explanation cannot be fully ruled out based on the present data. However, prior studies have shown that blind individuals can and do learn about colors as well as other purely visual phenomena (e.g. sparkle) (Shepard \& Cooper 1992; Marmor 1978; Saysani, Corballis \& Corballis, 2018; Bedny et al., under review). As noted in the introduction, Landau and Gleitman (1985) showed that Kelli, a blind 4-year-old, knew that colors are physical properties perceptible only with the eyes. Therefore, it is not the case that blind individuals do not learn anything about color. Rather, it seems that the colors of objects, and animal colors in particular, are less likely to be shared across blind and sighted individuals. These results raise doubts about the idea that blind individuals learn about what things look like primarily from sighted people's descriptions of appearance.

Instead, we hypothesize that blind individuals use folk taxonomy and other knowledge about animals (e.g. their habitat) to make inferences about what animals look like. Such inference works less well for color than it does for shape and texture because color is less inferentially-related to other properties of animals (e.g. their taxonomic category and behavior). This hypothesis is 
supported by the disagreement patterns that arose between the sighted and blind groups across the dimensions tested.

For blind, but not sighted participants, sorting based on color was correlated with taxonomy. The lack of correlation in the sighted is presumably because sighted people learn the colors of common animals from vision, and color and folk taxonomy are not in fact correlated (e.g. polar bears, swans, and sheep are all white). Blind individuals also used broad taxonomic distinctions to judge shape and texture. Blind people's judgments were more correlated across dimensions than those of the sighted. As noted above, there was greater agreement between blind and sighted participants about the appearance of broad folk taxonomic groups (e.g. birds and aquatic animals) than about animals within these groups (e.g. among mammals), with blind participants sometimes collapsing perceptual sub-groups made by the sighted within a taxonomic class. For example, when sorting by shape, blind participants tended to group all land mammals together, while sighted participants separated gorilla, polar bear, grizzly, panda, and sloth from the other quadrupeds (e.g. deer, rhino, lion). Blind people were no more likely than the sighted, however, to group birds with mammals or to put sea-dwelling animals with land animals. Similarly during texture sorting, blind participants grouped all land mammals together, whereas the sighted made distinctions within them, such as between short-haired animals (e.g. deer, zebra, and giraffe) and those with fur (e.g. grizzly, gorilla, and mammoth). Analogously, in the texture feature choice task, sighted participants distinguished among mammals whose skin is covered with fur (e.g. foxes and bears) and those that are hairless (e.g. hippos and elephants). By contrast, a sizable subset $(>30 \%)$ of blind participants responded that hippos, elephants, rhinos and pigs have fur. Blind participants were no more likely than the sighted to say that a bird had fur or skin, however. Blind participants were more likely than the sighted to report that sharks have scales (68\% vs. $20 \%)$, which is what one would infer from the fact that they are fish. By contrast, the majority of sighted participants reported that sharks have skin. (Sharks have fine scales that are difficult to discern through visual perception.)

Perhaps the best evidence for the idea that blind individuals relied on taxonomy to infer appearance comes from the odd-one-out shape task. When animal kind was pitted against appearance, sighted participants went with appearance, whereas the blind group tended to go with taxonomy (e.g. for parrot-ostrich-giraffe, a majority of sighted participants picked parrot as the odd-one-out, whereas blind participants chose giraffe; for wolf-gorilla-bear, sighted picked wolf, blind picked gorilla). Together, this evidence suggests that blind individuals use their knowledge of folk taxonomic distinctions, as well as other semantic information about animals (e.g. whether they live on land or in water) to make inferences about their appearance.

Humans across cultures organize animals into taxonomies and use these to make inferences about the physical and non-physical properties of animals. Animals belonging to the same taxa are judged to share characteristics, such as how they behave, grow, and catch diseases (e.g., Medin \& Atran, 2004, Lopez et al., 1997). Children and adults also use knowledge about what category an animal belongs to, to make inferences about its unobservable properties, such as its insides (e.g. Medin \& Ortony, 1989, Gelman \& Wellman, 1991). Analogously, individuals who are blind infer the surface appearance of an animal from an understanding of the category to which the animal belongs and of the way that category predicts surface properties (e.g. birds fly, all birds are likely to have a "winged" shape). Interestingly, although blind participants inferred color from taxonomy, they were less likely to do so than for shape and texture, suggesting that like the sighted, blind individuals are sensitive to the fact that color is less predictive of kind than shape (Landau, Smith \& Jones, 1992; Diesendruck \& Bloom, 2003). 
The primary way in which language transmits information about appearance is indirect. Language transmits information about kind and about how kind is related to surface features (e.g. ostriches are birds, and birds have wings and feathers), and the rest is left to the inferential mechanisms shared by sighted and blind people alike (Schulz, 2008; Williams, Lombrozo \& Rehder, 2013; Gelman \& Wellman, 1991; Gopnik \& Metzoff, 1997).

\section{How different is the knowledge of people who are blind and sighted? Some caveats}

The present study focused on knowledge of appearance and, in doing so, only scratched the surface of people's knowledge about animals. For example, although the present data suggest that blind and sighted people share folk taxonomic knowledge, the experiments were not designed to test it. In future work it would be interesting to ask whether there are any differences in folk taxonomies across people who live in the same culture but have different access to visual and linguistic sources. In some cases, we might expect vision to provide useful information about taxonomy. For example, watching lions hunt might tell us that they are related to other carnivores, and their shape provides clues about their relationship to other cats. In other cases, vision might be misleading, causing people to believe that evolutionarily distinct animals are similar (e.g. dolphins and sharks). Such differences in access may lead to intriguing taxonomic disagreements.

There is also other knowledge about animals that is almost certainly common to people who are sighted and blind that was not measured in the current study. Previous research has shown that even young children reason about animals in causal ways that go well beyond what they look like. For example, young children know that animals breathe, have internal invisible parts (e.g. hearts), have babies, and can hear and see things (e.g., Johnson \& Carey, 1998; Keil, 1989). Between the ages of 6 and 12, children develop more sophisticated concepts about life (e.g. that things can be alive but inanimate), death, "people-as-one-animal-among-many", and species kind (Johnson \& Carey, 1998). Blind and sighted adults almost certainly share such knowledge, which we did not test.

It is also possible that appearance-related knowledge is less shared for animals than for other categories of objects among blind and sighted people. It has been previously noted that people living in industrialized societies have rather impoverished knowledge of animals relative to societies living in closer contact with nature (Lopez et al., 1997; Atran, 1998; Atran et al., 2001). For example, Itzaj Maya people have different and richer representations of plant and animal life than American undergraduates living in Michigan (Lopez et al., 1997). Blind and sighted individuals living in industrialized societies might therefore be more likely to share appearancerelated knowledge of more ecologically relevant objects. Consistent with this idea, Landau \& Gleitman (1985) reported that Kelli knew the colors of some familiar objects (e.g. that her dog was golden, milk is white, and grass is green). Blind adults may also be more likely to agree with the sighted about the colors of common fruits and vegetables than the colors of "common" animals (see Connolly et al., 2007). Nevertheless, although the degree of shared object color knowledge may increase with ecological relevance and sheer frequency, we hypothesize that the general principles observed in the current study will still apply: individuals who are blind will be most likely to agree with the sighted about those aspects of appearance that are easiest to predict based on intuitive theories of how appearance works. 


\section{METHODS}

\section{Participants}

20 congenitally blind $(16 \mathrm{~F} / 4 \mathrm{M})$ and 20 control sighted $(13 \mathrm{~F} / 7 \mathrm{M})$ participants took part in the study. Control participants were matched to blind participants on age (blind group: $M=30$ years of age, $\mathrm{SD}=9$; sighted group: $\mathrm{M}=30, \mathrm{SD}=9$ ) and years of education (blind group: $M=16, S D=2$; sighted group: $M=15, S D=2$ ). One additional blind participant (participant 21) was dropped because they turned out to not be a native English speaker upon further screening. Most participants completed all of the experimental tasks. However, the following sorting tasks were only completed by a subset of blind participants: object sorting $(n=19)$, habitat sorting $(n=15)$, skin texture sorting $(n=19)$, shape odd-one-out $(n=18)$, texture feature choice $(n=19)$. The cause of blindness for all blind participants was due to damage anterior to the optic chiasm. To assess general cognitive abilities, the Woodcock Johnson III Tests of Achievement (subtests Word ID, Word Attack, Synonyms, Antonyms, Analogies; Woodcock et al., 2001) and letter span test (forward and backwards) were administered. All participants scored within two standard deviations from the mean of the current group on the WJIII tests. Participants were additionally recruited from Amazon's Mechanical Turk for height card ordering $(n=10)$, size card ordering $(n=10)$, and shape odd-one-out tasks $(n=15)$.

\section{Materials \& Methods}

Blind and sighted participants were we asked about the size (card ordering), height (card ordering), shape (card sorting and odd-man-out tasks), skin texture (card sorting and feature choice tasks), and color (card sorting) of animals (Fig. 1). As control conditions, we also asked about where household objects are stored (card sorting), as well as about the habitat and diet of animals (card sorting). Participants additionally rated their familiarity with all animals presented during the task (102 animals) on a scale of 1 to 4 .

\section{Card Ordering Task (Size and Height)}

Participants were asked to order a list of either 13 (size) or 15 (height) animals. Multiple lists of animals were first piloted on MTurk (i.e. participants typed in the order of animals on list of slots). Various lists were tested to ensure that the ordering was neither too easy nor too difficult. The final orderings (Fig. 3) were based on the average rankings for each item across MTurk participants' (e.g. for mosquito, most participants gave a rank of '1' but a few also responded '2', resulting in a ranking average of 1.3). Individual MTurk participants' agreement with the group ranking was $89.2 \%$ for size $(S D=12.7 \%)$ and $66 \%$ for height $(S D=15.8 \%)$, where agreement was calculated as the percentage of animals that were given the same rank.

Participants were instructed to order the animals first based on their size (i.e. "the overall amount of space that an animal takes up in the world") and their height (i.e. "the distance from the ground to the highest point of the animal when it is typical posture. For instance, a snake would typically have its whole body flat on the ground, so you are thinking about the distance from the ground to the top of its head when it is lying down, whereas a crocodile usually lies flat on its stomach, and for other four-legged animals like dogs, think about their height when they are standing on all fours"). 
For blind participants, animal or object names were written in Braille (contracted, Grade II) on small cardboard cards (around 3x1 inch in size), while names were written in print for sighted participants. At the start of the task, the experimenter placed all 13 (size)/15 (height) cards vertically in one of two predetermined orders (counterbalanced) and instructed the participants to read the animal cards out loud, starting from the top. Participants then started ordering the cards by picking the animal that they thought was the smallest/shortest. The experimenter then placed the chosen animal on the first slot of a long, felt board placed next to the vertically arranged animal cards (Fig. 1) and asked the participant to continue (i.e. pick the next smallest/shortest animal, and so on). At any time, participants could re-read and change the ordering of the cards on the board. Once all animal cards had been placed on board, the experimenter read the final list out loud to make sure participants were satisfied with the ordering. In-lab sighted and blind participants' rankings were compared against the average MTurk rankings.

\section{Card Sorting Task (Shape, Skin Texture, Color, Habitat, Diet, Objects)}

In the card sorting task, participants sorted the same 30 animal-cards into groups according to various sorting rules. The animals were selected as to maximize differential sorting across rules. There were 5 different animal sorting rules, 3 related to perceptible physical features (shape, skin texture and color) and the remaining 2 to habitat, and diet. The latter two were included as controls. Participants also sorted 29 objects according to where they are stored around the house, as a practice and control.

Participants were informed that they would sort the same cards multiple rounds, each time according to a different rule, with no restrictions on the number of groups that can be formed. At the start of each sorting, the experimenter handed the cards to the participants one at a time, reading them out loud. Cards had Velcro taped to the back such that blind participants could easily place and remove them from a felt board which served as their sorting surface. Participants were free to re-read and adjust their groups at any time. At the end of each sorting, participants were asked to provide a verbal label to describe each group ("How would you describe this group?"). To ensure that participants were satisfied with their groupings, the experimenter read the label provided by the participant, along with the members of the group, out loud (e.g., "In this group that you labeled 'Kitchen', you have spoon, bowl, fork, and spatula”).

The object sorting task was administered first and served as a practice round to get participants accustomed to the task. Before each sorting round, participants were given the specific rule (e.g. "Sort in different groups according to where these objects are usually stored"). The experimenter then instructed the participants to sort the animals according to 1) their shape ("the outline of their body"); 2) the texture of their skin; 3) their color or pattern; 4) the habitat ("where they live"); and 5) their diet ("what they eat"). All participants performed the task in this fixed order. Before the start of the animal sorting tasks, participants were further reminded that some animals may fall into different groups depending on the specific individual of the species (e.g. dogs can have many different shapes), and to decide based on their intuition of the most common kind of a given animal (e.g. a Labrador).

\section{Card Sorting Task Similarity Matrix Analysis}

A similarity matrix was constructed for each participant and each sorting round, assigning, for any given pair of objects or animals, ' 1 ' if the two items had been placed in the same group and ' 0 ' 
otherwise. The matrices of all participants were averaged to obtain one group matrix per group (Fig. 4 and 5). The two group matrices were then correlated. To examine variability within each group, each individual blind or sighted participants' matrix was correlated to the average of the group with the single individual left out (within-group, sighted-individual-to-sighted-group or blindindividual-to-blind-group, S-S and B-B). In addition, each individual blind participant's matrix was correlated with the sighted group matrix (across group, blind-individual-to-sighted-group, B-S). Spearman correlation was used for all matrix correlations, and Fisher's z-transformation was applied to the resulting rank correlation coefficients (rho) to allow comparison across groups.

We examined similarity across sorting rules within each group (e.g., shape vs. color for blind group) as well as similarity across groups within each sorting rule (e.g., blind vs. sighted for shape) (Fig. 4). For within-group correlations, the group matrices for shape, texture, and color were correlated with each other, resulting in three correlation coefficients. For across-group correlations, the blind group and sighted group matrices were correlated for each sorting rule.

To examine the degree to which participant sorting was predictable based on taxonomic similarities between animals, each group matrix, for each sorting round, was further correlated with an evolutionary distance similarity matrix (Fig. 4). Evolutionary distance between pairs of animals was obtained using an online database pooling published data from 2000 studies of species diversification (timetree.org, Hedges et al., 2015). To enable statistical comparison of correlations with taxonomy across blind and sighted groups, each individual participants' similarity matrix was additionally correlated with the evolutionary distance matrix and z-transformed (SI Fig. 2).

\section{Sorting Pile Descriptions Analysis}

During the card sorting task, participants were asked to label each of the piles they created. For each sorting rule (i.e. shape, texture, or color) and each animal, a verbalizability (or codability) score was calculated using Simpson's Diversity Index, as below, where for unique words 1 to $R$ provided for each animal, $\mathrm{n}$ is the count of how many times each word was used across participants, and $\mathrm{N}$ is the total number of words (Majid \& Burenhult, 2014; =; Majid et al., 2018). The index ranges from 0 to 1 , where a score of 0 would indicate that the same word was never used more than once (i.e. low verbalizability), and 1 would suggest that all participants provided the same word (i.e. high verbalizability).

$$
D=\frac{\sum_{i=1}^{R} n_{i}\left(n_{i}-1\right)}{N(N-1)}
$$

All words provided by participants were treated as discrete utterances, with the following exceptions, which were treated as single words: upside-down, on the ground, and any "is-like" statement (e.g. fish-like, human-like).

\section{Odd-One-Out Triplets Task (Shape)}

During the shape triplets task, participants were presented with three animals at a time and asked to choose the animal that is different from the other two based on overall shape. The task was administered verbally for both blind and sighted participants. Participants were reminded to ignore 
other features such as color, size, texture, or type of animal. Triplets of animals were picked based on piloting on MTurk and created to minimize confounds with non-shape dimensions like size and category (e.g. 'wolf, bear, and gorilla' and 'parrot, giraffe, ostrich', Fig. 6). Answers were scored as correct or incorrect based on the answer agreed on by the MTurk group.

\section{Feature Choice Task (Texture)}

To further probe knowledge about the texture of animals, participants performed a feature choice task. The task was administered verbally for both blind and sighted participants. The experimenter read out loud the name of 30 animals (Fig. 6) one at a time, and participants had to decide whether the animal had scales, feathers, skin, or fur. If 'skin' was picked, participants were further asked whether the skin is smooth and rough, and for 'fur', whether the fur is short, medium, or long in length and whether it is thick or fine. The 30 animals used for this task were different from the 30 animals used in the sorting task. For a majority of the 30 animals, there is a clear correct answer: birds have feathers, reptiles and fish have scales, and some animals have no hair or fur (e.g. worm, frog, toad, dolphin). Animals that have skin with some short hairs or bristles (e.g. pig) were scored as correct for 'skin' responses, and animals that have fur in some but not all parts of the body (e.g. horse, donkey) were scored correct if 'fur' was the answer.

The data, processing code, and analysis scripts reported in this paper will be deposited in a GitHub repository, https://github.com/judyseinkim/Animals.

\section{Acknowledgements:}

We thank the participants, the blind community, and the National Federation of the Blind for making this research possible, Lindsay Yazzolino for insights that served as motivation for this project, and Erin Brush for assistance with data collection. We are grateful to Justin Halberda, Jon Flombaum, Chaz Firestone, and Barbara Landau for their astute feedback on data interpretation.

\section{REFERENCES}

1. Scamander, N., \& Rowling, J. K. (2001). Fantastic beasts and where to find them.

2. Locke, J. (1924). 1690. An essay concerning human understanding, 1.

3. Berkeley, G. (1709). An essay towards a new theory of vision.

4. Hume, D. (1758). Essays and treatises on several subjects.

5. Landau, B., \& Gleitman, L. (1985). Language and Expirience.

6. Lenci, A., Baroni, M., Cazzolli, G., \& Marotta, G. (2013). BLIND: a set of semantic feature norms from the congenitally blind. Behavior research methods, 45(4), 1218-1233.

7. Shepard, R. N., \& Cooper, L. A. (1992). Representation of colors in the blind, color-blind, and normally sighted. Psychological Science, 3(2), 97-104.

8. Marmor, G. S. (1978). Age at onset of blindness and the development of the semantics of color names. Journal of experimental child psychology, 25(2), 267-278.

9. Saysani, A., Corballis, M. C., \& Corballis, P. M. (2018). Colour envisioned: concepts of colour in the blind and sighted. Visual Cognition, 26(5), 382-392.

10. Connolly, A. C., Gleitman, L. R., \& Thompson-Schill, S. L. (2007). Effect of congenital blindness on the semantic representation of some everyday concepts. Proceedings of the National Academy of Sciences, 104(20), 8241-8246.

11. Gleitman, L. R., Cassidy, K., Nappa, R., Papafragou, A., \& Trueswell, J. C. (2005). Hard words. Language Learning and Development, 1(1), 23-64. 
12. Majid, A., Roberts, S. G., Cilissen, L., Emmorey, K., Nicodemus, B., O'Grady, L., Woll, B., LeLan, B., de Sousa, H., Cansler, B. L., Shayan, S., de Vos, C., Senft, G., Enfield, N. J., Razak, R. A>, Fedden, S, Tufvesson, S., Dingemanse, M., Ozturk, O., Brown, P., Hill, C., Le Guen, O., Hirtzel, V., van Gijn, R., Sicoli, M. A., \& Levison, S. C. (2018). Differential coding of perception in the world's languages. Proceedings of the National Academy of Sciences, 115(45), 11369-11376.

13. Winter, B., Perlman, M., \& Majid, A. (2018). Vision dominates in perceptual language: English sensory vocabulary is optimized for usage. Cognition, 179, 213-220.

14. Van Deemter, K. (2009). Utility and language generation: The case of vagueness. Journal of Philosophical Logic, 38(6), 607.

15. Solt, S. (2015). Vagueness and imprecision: Empirical foundations. Annu. Rev. Linguist., 1(1), 107-127.

16. Landau, B., \& Jackendoff, R. (1993). Whence and whither in spatial language and spatial cognition?. Behavioral and brain sciences, 16(2), 255-265.

17. Fussell, S. R., \& Krauss, R. M. (1992). Coordination of knowledge in communication: Effects of speakers' assumptions about what others know. Journal of personality and Social Psychology, 62(3), 378.

18. Westerbeek, H., Koolen, R., \& Maes, A. (2015). Stored object knowledge and the production of referring expressions: the case of color typicality. Frontiers in psychology, 6, 935.

19. Talmy, L. (1983). How language structures space. In Spatial orientation (pp. 225-282). Springer, Boston, MA.

20. Pinker, S. (2007). The stuff of thought: Language as a window into human nature. Penguin.

21. Majid, A., \& Burenhult, N. (2014). Odors are expressible in language, as long as you speak the right language. Cognition, 130(2), 266-270.

22. Levinson, S. C., \& Majid, A. (2014). Differential ineffability and the senses. Mind \& Language, 29(4), 407427.

23. Hedges, S. B., Marin, J., Suleski, M., Paymer, M., \& Kumar, S. (2015). Tree of life reveals clock-like speciation and diversification. Molecular biology and evolution, 32(4), 835-845.

24. Rubinstein, D., Levi, E., Schwartz, R., \& Rappoport, A. (2015). How well do distributional models capture different types of semantic knowledge? In Proceedings of the 53rd Annual Meeting of the Association for Computational Linguistics and the 7th International Joint Conference on Natural Language Processing (Volume 2: Short Papers) (Vol. 2, pp. 726-730).

25. Collell, G., \& Moens, M. F. (2016). Is an image worth more than a thousand words? on the fine-grain semantic differences between visual and linguistic representations. In Proceedings of COLING 2016, the 26th International Conference on Computational Linguistics: Technical Papers (pp. 2807-2817).

26. He, C., Peelen, M. V., Han, Z., Lin, N., Caramazza, A., \& Bi, Y. (2013). Selectivity for large nonmanipulable objects in scene-selective visual cortex does not require visual experience. Neuroimage, 79, 1-9.

27. Mahon, B. Z., Anzellotti, S., Schwarzbach, J., Zampini, M., \& Caramazza, A. (2009). Category-specific organization in the human brain does not require visual experience. Neuron, 63(3), 397-405.

28. Peelen, M. V., He, C., Han, Z., Caramazza, A., \& Bi, Y. (2014). Nonvisual and visual object shape representations in occipitotemporal cortex: evidence from congenitally blind and sighted adults. Journal of Neuroscience, 34(1), 163-170.

29. Kitada, R., Okamoto, Y., Sasaki, A. T., Kochiyama, T., Miyahara, M., Lederman, S. J., \& Sadato, N. (2013). Early visual experience and the recognition of basic facial expressions: involvement of the middle temporal and inferior frontal gyri during haptic identification by the early blind. Frontiers in human neuroscience, $7,7$.

30. van den Hurk, J., Van Baelen, M., \& de Beeck, H. P. O. (2017). Development of visual category selectivity in ventral visual cortex does not require visual experience. Proceedings of the National Academy of Sciences, 114(22), E4501-E4510.

31. Bi, Y., Wang, X., \& Caramazza, A. (2016). Object domain and modality in the ventral visual pathway. Trends in cognitive sciences, 20(4), 282-290.

32. Bull, R., Rathborn, H., \& Clifford, B. R. (1983). The voice-recognition accuracy of blind listeners. Perception, 12(2), 223-226.

33. Cuevas, I., Plaza, P., Rombaux, P., De Volder, A. G., \& Renier, L. (2009). Odour discrimination and identification are improved in early blindness. Neuropsychologia, 47(14), 3079-3083.

34. Wong, M., Gnanakumaran, V., \& Goldreich, D. (2011). Tactile spatial acuity enhancement in blindness: evidence for experience-dependent mechanisms. Journal of Neuroscience, 31(19), 7028-7037.

35. Medin, D. L., \& Atran, S. (2004). The native mind: biological categorization and reasoning in development and across cultures. Psychological review, 111(4), 960.

36. Lopez, A., Atran, S., Coley, J. D., Medin, D. L., \& Smith, E. E. (1997). The tree of life: Universal and cultural features of folkbiological taxonomies and inductions. Cognitive psychology, 32(3), 251-295.

37. Medin, D. L., \& Ortony, A. (1989). Psychological essentialism. Similarity and analogical reasoning, 179, 195.

38. Gelman, S. A., \& Wellman, H. M. (1991). Insides and essences: Early understandings of the nonobvious. Cognition, 38(3), 213-244. 
39. Landau, B., Smith, L. B., \& Jones, S. (1992). Syntactic context and the shape bias in children's and adults' lexical learning. Journal of Memory and Language, 31(6), 807-825.

40. Diesendruck, G., \& Bloom, P. (2003). How specific is the shape bias?. Child development, 74(1), $168-178$.

41. Schulz, L. E., Standing, H. R., \& Bonawitz, E. B. (2008). Word, thought, and deed: The role of object categories in children's inductive inferences and exploratory play. Developmental Psychology, 44(5), 1266.

42. Williams, J. J., Lombrozo, T., \& Rehder, B. (2013). The hazards of explanation: Overgeneralization in the face of exceptions. Journal of Experimental Psychology: General, 142(4), 1006.

43. Gelman, S. A., \& Wellman, H. M. (1991). Insides and essences: Early understandings of the nonobvious. Cognition, 38(3), 213-244.

44. Gopnik, A., \& Meltzoff, A. N. (1997). Learning, development, and conceptual change.

45. Johnson, S. C., \& Carey, S. (1998). Knowledge enrichment and conceptual change in folkbiology: Evidence from Williams syndrome. Cognitive Psychology, 37(2), 156-200.

46. Keil, F. C. (1992). The origins of an autonomous biology. In Modularity and constraints in language and cognition: The Minnesota symposia on child psychology (Vol. 25, pp. 103-137). Hillsdale, NJ: Erlbaum.

47. Atran, S. (1998). Folk biology and the anthropology of science: Cognitive universals and cultural particulars. Behavioral and brain sciences, 21(4), 547-569.

48. Atran, S., Medin, D., Lynch, E., Vapnarsky, V., Ek, E. U., \& Sousa, P. (2001). Folkbiology doesn't come from folkpsychology: Evidence from Yukatek Maya in cross-cultural perspective. Journal of Cognition and Culture, 1(1), 3-42.

\section{FIGURE LEGENDS}

Fig. 1. Experimental tasks used to probe knowledge about size (card ordering), height (card ordering), shape (card sorting and odd-one-out), skin texture (card sorting and feature choice), and color (card sorting). Card tasks required participants to order or sort cards based on a given dimension. Names of animals (or objects) were written on cardboard cards, in Braille for blind and in print for sighted participants.

Fig. 2. Familiarity ratings (1: least familiar to 4 : most familiar) for all unique animals used across tasks $(n=102)$.

Fig. 3. Results for animal size and height (card ordering). Bar graphs show sighted and blind participants' overall agreement with rankings obtained from MTurk. Plots on the upper right show average rankings provided for each animal. Pairwise comparisons between successive animals are significant $(p<0.05)$ unless noted otherwise ('n.s.'). Rainbow plots show individual participants' rankings. Each column is a single participant, ordered from lowest to height agreement with average mTurk ranking (right-to-left). Error bars are mean +/- SEM.

Fig. 4. Correlations of sorting results (shape, skin texture, color) with taxonomy (evolutionary distance), as well as within-group and across-group correlations. All matrices are ordered based on the optimal order for evolutionary distance. Red indicates non-significant correlations (Spearman's rho).

Fig. 5. Results from card sorting task for animal shape, texture, and color. Lower rows of matrices show sorting similarity matrices averaged across participants within each group and sorting round (color indicates percentage of participants who grouped a given pair of animals into the same sorting pile). Bar graphs: correlation of individual participant to group similarity matrices, within group (sighted-individual-to-sighted-group and blind-individual-to-blind-group) and across group (blind-individual-to-sighted-group). Mean +/- SEM. Top row of matrices show similarity based on sighted participants' verbal labels of sorted piles. Within each sorting dimension, matrices are ordered according to the optimal order for sighted participants' sorting results. 
Fig. 6. Verbalizability of sighted participants' sorting group descriptions (color, texture, shape). Simpson's Diversity Index calculated for individual animals. Mean +/- SEM.

Fig. 7. A. Results for animal shape (odd-one-out triplets task). Bar graphs shows sighted and blind participants' overall agreement with MTurk participants. Box shows one example triplet, with the most common sighted group answer in red and most common blind group answer in blue. B. Results for skin texture (feature choice task). Bar graphs shows sighted and blind participants' overall agreement with MTurk participants. Greyscale figures show percentages of participants who chose scales, feathers, skin, or fur for all items. Error bars are mean +/- SEM. 


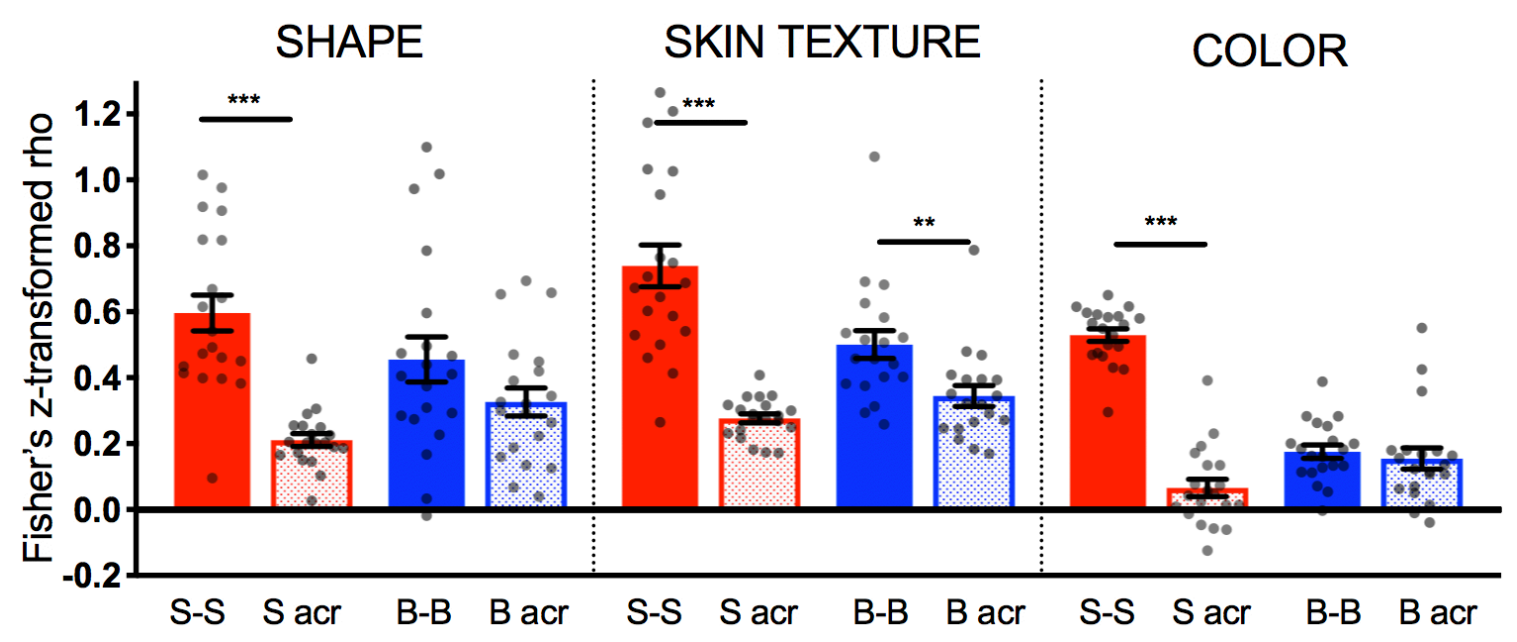

Fig. S1. Within- and across-dimension correlations (card sorting). Correlation coefficients for within- and across-dimensions (shape, skin texture, and color card sorting). Within-dimension correlation bars (S-S and B-B; individual-to-group, leave-one-out correlations) are reproduced from Fig. 5. Across-dimension correlations are average of individual participants' correlations with the other two dimensions (e.g., for shape, average of shape-to-texture and shape-to-color correlations). Mean +/- SEM. 


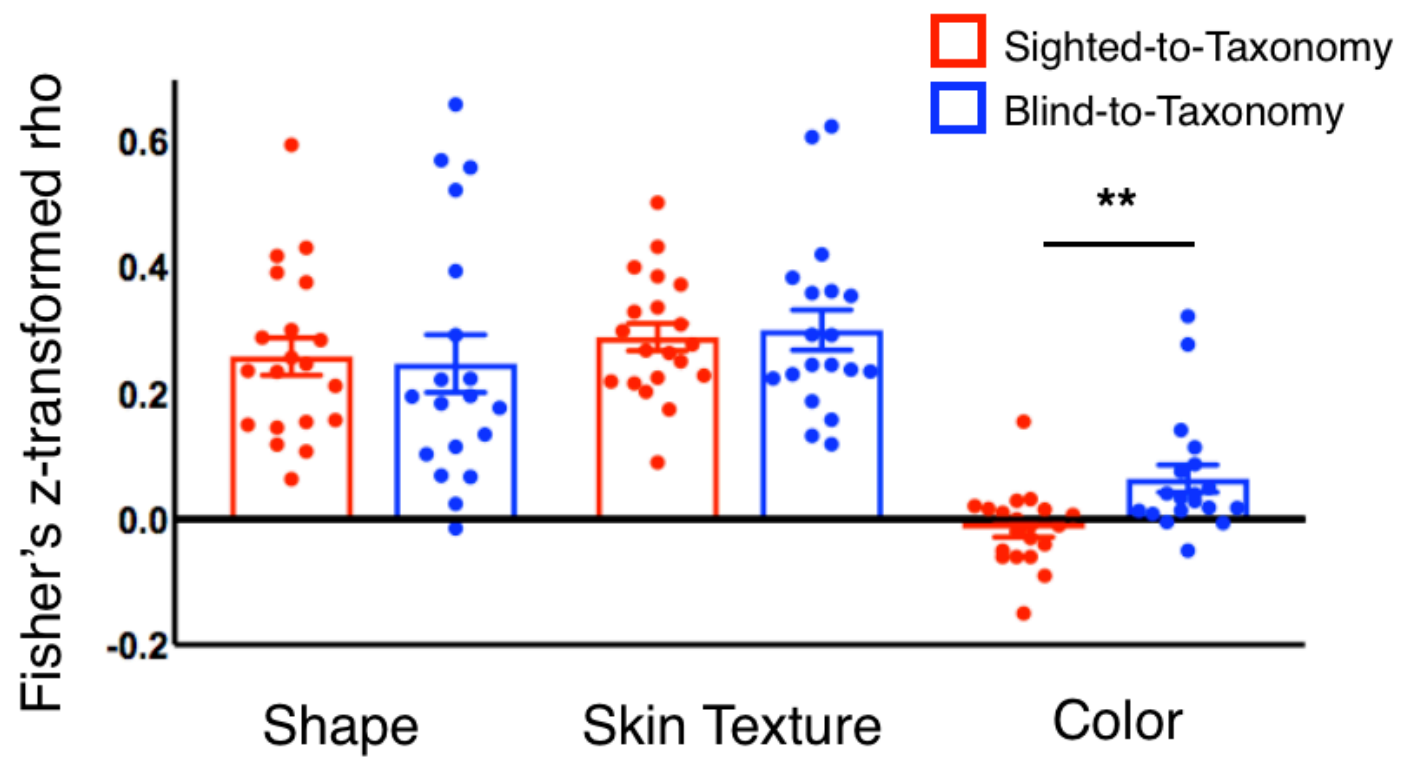

Fig. S2. Correlations with taxonomy (card sorting). Sorting results correlated with taxonomy (evolutionary distance). Bars show fisher's z-transformed correlation coefficients for correlation between individual participants' similarity matrix and taxonomy matrix. 


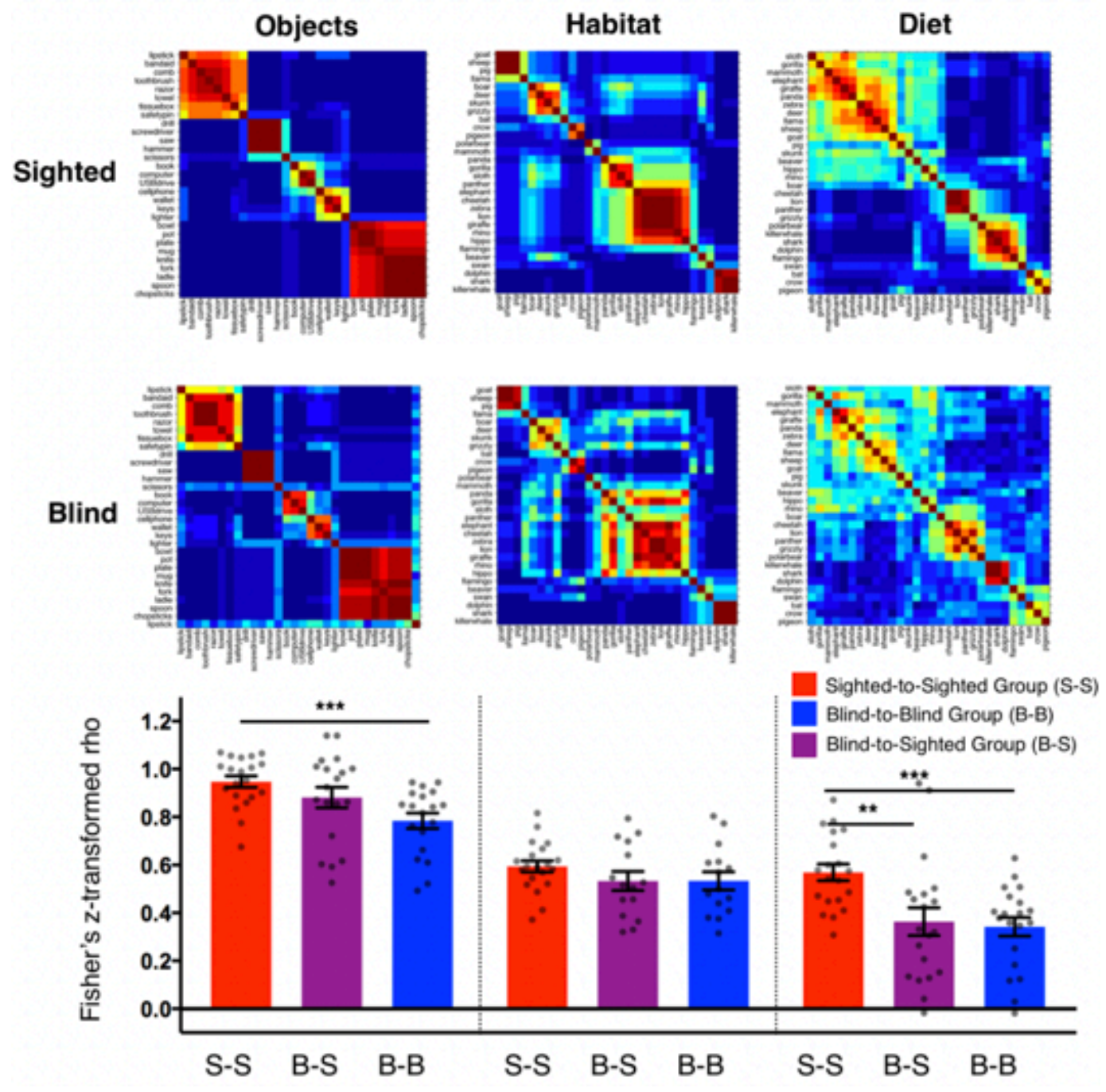

Fig. S3. Performance on control conditions (card sorting). Results from card sorting task for object (storage place), animal habitat, and diet. Similarity matrices averaged across participants within each group and sorting round (color indicates percentage of participants who grouped a given pair of animals into the same sorting pile). Animals are ordered based on optimal ordering of the sighted group, based on hierarchical clustering, for better visualization. Bars: correlation of individual participant to group within group (sighted-individual-to-sighted-group and blindindividual-to-blind-group) and across group (blind-individual-to-sighted-group). Mean +/- SEM. 